\title{
Evaluation of VoIP Performance in Downlink Cellular Networks With Multihop Relaying
}

\author{
Nikolaj Marchenko*, Jakob Hoydis*, Aik Chindapol* and Rainer Schoenen ${ }^{+}$ \\ ${ }^{*}$ Siemens Corporate Research, Princeton, NJ \\ \{nikolaj.marchenko, jakob.hoydis\}@rwth-aachen.de, aik@ieee.com \\ ${ }^{+}$Chair of Communication Networks (ComNets), FB6, RWTH Aachen University, Germany \\ rsecomnets.rwth-aachen. de
}

\begin{abstract}
We analyze the impacts of multi-hop relaying on the quality of service of voice transmission in the downlink OFDMA communication system. It is shown that the system performance in terms of capacity and quality strongly depends on deployment and operating parameters such as the location of relays, traffic loads and the allowable packet error rate. The impact of relaying is less evident when the traffic load is light and the base station can effectively cover the whole cell. In the high traffic load region, multi-hop relaying is most effective in reducing the outage probability while increasing the cell throughput.
\end{abstract}

\section{Introduction}

A beyond 3G cellular system envisages ubiquitous broadband wireless access with support for fixed, nomadic and fully mobile operations while offering a vast spectrum of voice, video and data services. To achieve this, very high data rates with comprehensive Quality-of-Service (QoS) control mechanisms are necessary. However, unlike traditional voice users, where uniform voice quality is expected and delivered throughout the cell, data users experience dramatically different data rates depending on where they are in the cell [1] due to the fluctuation of the desired signal and interference. In addition, the proposed spectrum for next generation wireless broadband networks is located well above the $2 \mathrm{GHz}$ band and the signal quality at the receiver is much more affected by the propagation loss, especially in non-line-of-sight (NLOS) conditions. Increasing the base station (BS) density could alleviate this problem; however, it incurs prohibitive maintenance and infrastructure costs. Alternatively, while maintaining the same BS density, a scheduler may allocate more resources to users who do not have good signal coverage to smooth out the perceived data rate at the cost of lower spectral efficiency and system throughput. Another approach, first introduced in [2], [3], is the use of relay stations (RS), which are intermediate communication nodes that receive and forward data to the destination.

The primary benefit of multi-hop relaying comes from the reduction of the overall path loss between the source and the receiver and thus improving the Signal-to-Interferenceplus-Noise-Ratio (SINR). This is especially important in heavily shadowed urban areas where the link between either the source and the relay or the destination and relay can be significantly better than the direct link. However, the penalty for relaying is additional overhead due to transmissions of data over multiple hops and higher interference. Therefore, multi-hop relaying requires careful system design and network planning in order to obtain optimal benefit in resource utilization and QoS-specific performance.

There have been extensive studies about the performance analysis of multi-hop relaying in wireless cellular networks. In [1], a brief history and benefits of the relay concept, including cooperative relaying, virtual antenna arrays, routing and radio resource management, as well as an outlook to future research areas are presented. The authors in [4] analyze the throughput enhancement for downlink cellular networks and conclude that most of the performance gain can be obtained with two to three relay hops and it can be converted into improvement of QoS. It is also noted that relaying not only increases throughput but also provides fairness among users, since QoS is more equally distributed within the cell. The authors in [5] study the effect of fixed relays on throughput, system capacity, spectral efficiency and delay using HiperLAN2. In this paper, relays are used to extend cell size and achieve coverage in shadowed urban areas. In [6], the authors analyze the ability of the IEEE 802.16e system to simultaneously manage traffic with strict QoS requirements, such as VoIP, Video on Demand, online gam- 
ing and data applications. They study the effect of different frame durations on delay and throughput and the increase in overhead for a growing number of users. In [7], [8], the authors investigate performance of VoIP-related QoS mechanisms of IEEE 802.16 for one-hop transmissions.

In this paper, we study the impact of multi-hop relaying deployed to enhance the capacity on QoS specific to VoIP. The IEEE 802.16 system is used as a model for our analysis. The rest of the paper is structured as follows: Section II explains the QoS mechanisms in 802.16. In Section III we give a detailed description and explanation of our system model. Section IV shows results and a performance analysis. Conclusions are drawn in Section V.

\section{QoS in IEEE 802.16}

There is a need for the system to satisfy the requirements of many users with different services simultaneously in order to guarantee satisfactory experience of users. Each of the different data streams has its individual requirements to transmission rate, delay, jitter and packet-loss and has a different optimum packet size. In IEEE 802.16 [9], each application service is characterized by a unique service flow that includes sets of corresponding QoS parameters. The standard also provides five different scheduling classes that are designed to guarantee packets transmission according to QoS requirements for a wide scope of applications.

VoIP traffic in IEEE 802.16 is managed by either the Unsolicited Grant Service (UGS), Real-Time Polling Service (rtPS) or Extended Real-Time Polling Service (ertPS) scheduling classes. The UGS class is designed to support real-time data streams with a fixed packet size and periodic transmission intervals suitable for a constant bit rate coded such as G.711. The Base Station (BS) grants service periodically to the Mobile Station (MS) to ensure a constant transmission rate. The rtPS and ertPS classes are especially optimized for VoIP traffic with variable bit rates. Since voice traffic is sensitive to delay, jitter and packet loss, the QoS parameters of IEEE 802.16 can be set up to allow audio packets to be delivered within the delay and error rate bounds. If a voice packet is delayed by time greater than the maximal latency, it is dropped out of the queue and the next packet is considered for the following transmission.

Other supported scheduling classes are Best-Effort Service (BE), used for applications without delay and rate requirements (e.g., web browsing), and Non-Real-Time Polling Service (nrtPS), used for applications that require guaranteed data rates, but are insensitive to delays (e.g., File Transfer Protocol, FTP) [9].

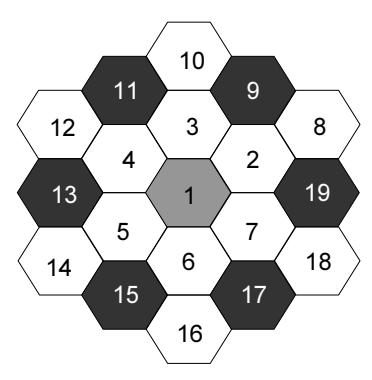

Figure 1. 19 cell network model

\section{System Description}

\subsection{General System Description}

We analyze a downlink communication between the base station, relay stations, and mobile stations in a 19 cell setup shown in Figure 1. Transmitters in surrounding cells cause interference to the receivers in the central cell, which is also referred to as the victim cell. Power control is not considered in this paper. In order to reduce the effects of inter-cell interference, a frequency re-use factor of three is used, meaning that three adjacent cells always have different transmission frequency sets. Cells causing interference to the victim cell are shaded in Figure 1. For the traffic source, we assume G.711 voice codec transmissions over the UGS service class. A $10 \mathrm{~ms}$ packet contains 80 bytes G.711 payload, 40 bytes of UDP/IP/RTP header and 6 bytes of IEEE 802.16 MAC header. The UGS mechanism guarantees that each user transmits 126 bytes of MAC PDU every $10 \mathrm{~ms}$. The resulting voice quality given by the R-value from scale of 0 to 100 is calculated according to the ITU E-model [10] as

$$
\begin{aligned}
R= & 94.2-0.024 \delta-0.11(\delta-177.3) H \\
& -\beta_{1}-\beta_{2} \log \left(1+\beta_{3} \psi\right),
\end{aligned}
$$

where $\delta$ is delay in ms, $\psi$ packet loss percentage, $H$ is set to 1 if the delay is higher than 177.3 and 0 otherwise. $\beta_{1}$, $\beta_{2}$ and $\beta_{3}$ are specific for the used voice codec.

\subsection{Propagation Models}

The propagation models are chosen from the WINNER II project [11] and correspond to a realistic urban network deployment scenario. Relay stations are $15 \mathrm{~m}$ high to guarantee LOS to the serving BS but NLOS to interfering BSs. BSs and MSs are located at the height of $25 \mathrm{~m}$ and $1.5 \mathrm{~m}$ respectively. We further assume a transmission frequency of $3.5 \mathrm{GHz}$, system bandwidth of $10 \mathrm{MHz}$ and transmission power for both $\mathrm{BS}$ and $\mathrm{RS}$ of $47 \mathrm{dBm}$. The pathloss for each link can be presented in the following form:

$$
P L(d)[d B]=A \cdot \log _{10}(d[m])+B,
$$




\begin{tabular}{lccc}
\hline Link & A & B & Scenario \\
\hline BS $\Leftrightarrow$ RS & 23.5 & 39.4 & B5a (LOS) \\
\hline Interfering BS $\Leftrightarrow$ RS & 23.5 & 54.4 & B5f (NLOS) \\
\hline BS/RS $\Leftrightarrow$ MS & 35.74 & 39.47 & C2 (NLOS) \\
\hline Interfering BS $\Leftrightarrow$ MS & 35.74 & 39.47 & C2 (NLOS) \\
\hline
\end{tabular}

Table 1. Propagation coefficients

where $d$ is the distance from transmitter to receiver in meters, $A$ and $B$ are coefficients specific for each propagation environment and system setup. Table 3.2 summarizes the chosen parameters for all relevant links.

\subsection{Interference Model}

The average level of interference coming from a particular BS depends on its traffic load. Due to the interference averaging nature of OFDMA, interference coming from a particular cell to the victim cell is averaged and proportional to the traffic load of that cell. The total interference at each mobile station $j$ in the victim cell is assumed to be static during the simulation time for both single-hop and multi-hop cases and can be calculated as the sum of received power from all $n$ interferers as

$$
I_{j}=\sum_{i=1}^{n} I_{i}\left(d_{i, j}\right),
$$

where $d_{i, j}$ is the distance between the $M S_{j}$ in the central cell and the interferer $i$ with power $I_{i}$ including the propagation loss of $P L\left(d_{i, j}\right)$.

Figure 2 shows principle structure of the OFDMA frame with two-hop transmission capability. Both DL and UL subframes are divided in two parts - for first-hop and secondhop transmissions respectively[12]. Since they are clearly separated from each other in time, there is no intra-cell interference between the BS and the RSs. We assume frame synchronization among all BSs. Thus, interfering BSs only have impact on the BS transmission and interfering RSs disturb only RSs transmissions in the victim cell. Interference to an RS-MS link within the victim cell comes from any RS from an interfering cell and it is difficult to identify which relay causes disturbance at any given time. Since there is no inter-cell coordination between relay nodes, any RS-MS transmission can be disturbed by various transmissions from neighboring RSs. Therefore, for a static environment, RS-generated interference from a particular cell at a mobile can be estimated by averaging interference of all RSs within that cell:

$$
I_{i}^{R S}(d)[W]=\frac{\sum_{k=1}^{N_{i}} I_{i}^{R S_{k}}\left(d_{i k}\right)}{N_{i}}[W],
$$

where $I_{i}^{R S}$ is the averaged interference coming from $N_{i}$ RSs in cell $i$. The interferences from all cells are summed

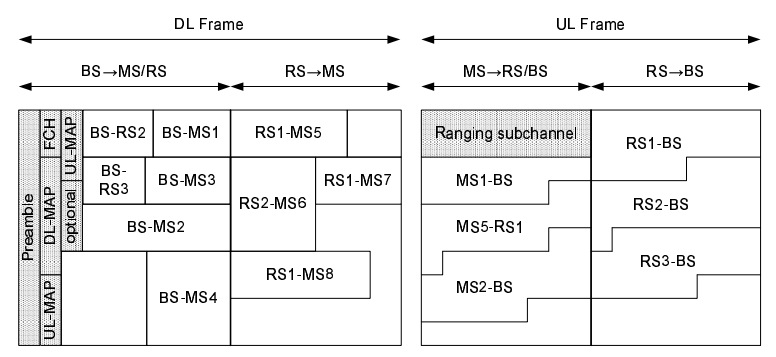

Figure 2. OFDMA frame structure with two-hop transmission capability [12]

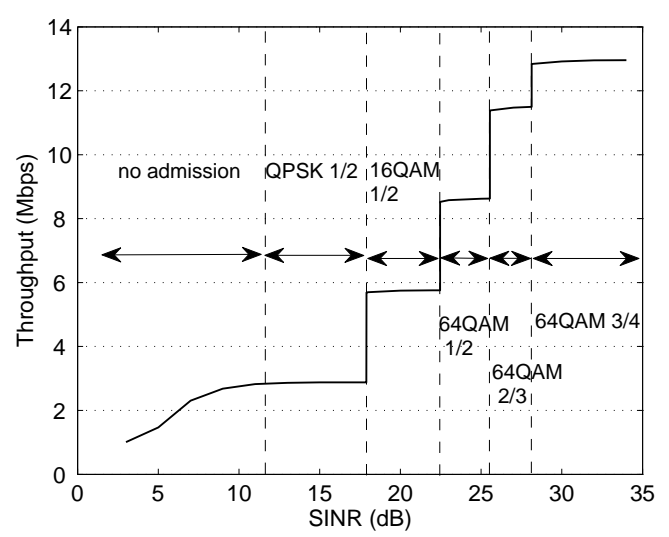

Figure 3. Max. achievable throughput at PER threshold of $1 \%$

up and the result gives the total average interference at the mobile in the victim cell as

$$
I_{R S}[W]=\sum_{i} I_{i}^{R S}[W] .
$$

The thermal noise at the receiver at room temperature is assumed to be $-104 \mathrm{dBm}$.

\section{Results and Analysis}

In this paper, we obtain the link-level results using the IEEE 802.16e system [13] with OFDMA, convolutional code, $5 \mathrm{~ms}$ frame size and $10 \mathrm{MHz}$ bandwidth. Figures 3 shows an example of the downlink link-level throughput vs. SINR over the ITU-R Ped B [14] channel model at $1 \%$ maximum PER and indicates the switching between modulation and coding schemes (MCS) that provide the maximal data rate.

Although the IEEE 802.16 standards specify 7 MCS levels that can be used, only MCSs that yield maximum throughput within the allowable PER are used in our calculation. In our simulation, uniformly distributed G.711 users 


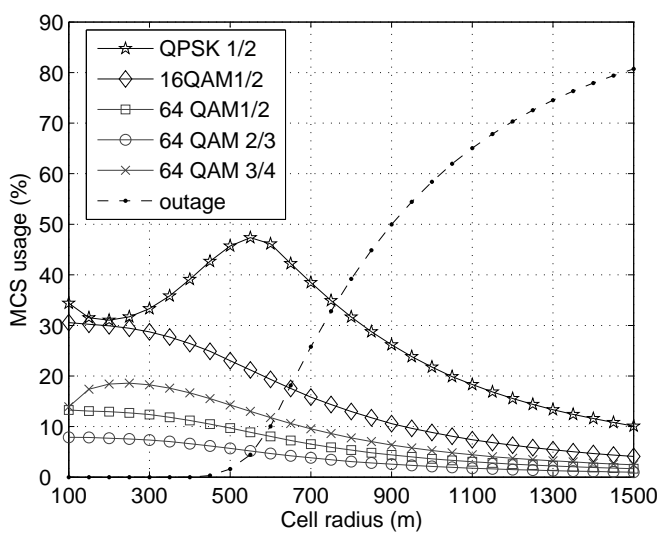

Figure 4. MCS usage in a non-relay cell

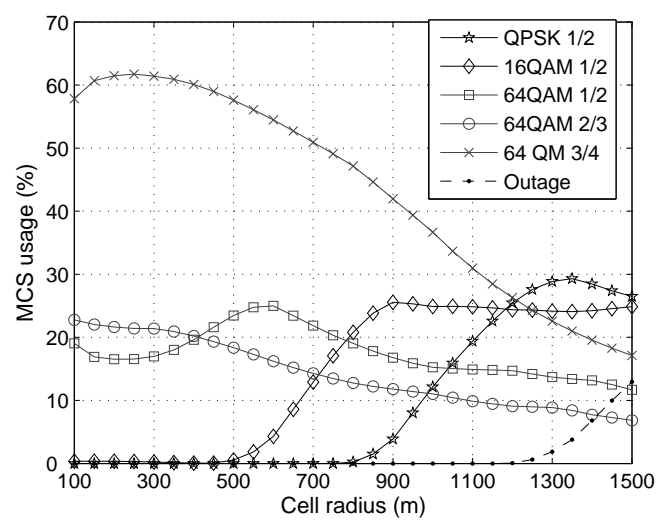

Figure 5. MCS usage in a cell with six relays

are randomly placed in the victim cell. The interference power is adjusted according to the traffic load. When relays are used, they are located halfway between the BS and the cell edge with equal spacing between relays.

Figure 4 shows the usage of different modulation schemes versus the cell radius. The maximum PER is fixed at $1 \%$, meaning that users operating at a higher PER either switch to the next lower modulation scheme or are blocked (outage) if they already use the most robust MCS, which is QPSK $1 / 2$. It is assumed that interfering cells have $50 \%$ traffic load. As the cell radius increases and the corresponding SINR degrades, the number of users applying QPSK 1/2 rapidly grows. Starting from a cell radius of $500 \mathrm{~m}$ the outage probability begins to rise quickly as the cell is not completely covered by the BS anymore. The growing percentage of outage leads to a decline in the number of served users.

Figure 5 also shows the usage of different modulation schemes in a cell with six RSs for the same system configuration. It can be seen that the most users experience

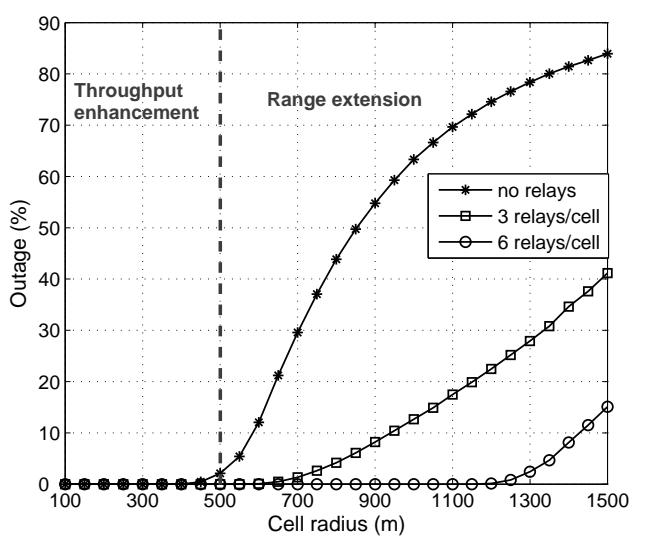

Figure 6 . Outage probability vs. cell radius

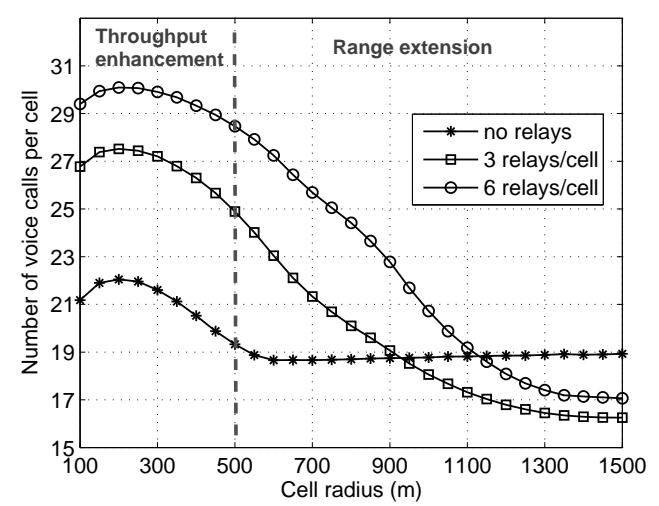

Figure 7. Number of voice calls vs. cell radius

much better SINR and are able to use higher MCSs than in a conventional cell. In Figure 6 we compare the outage probability over the cell radius for a conventional cell and a cell with three and six relays, which are located at the half of the cell radius from the BS. The maximum PER is fixed at $1 \%$. The benefit of relays becomes more relevant as we increase the cell coverage. Below the radius of $500 \mathrm{~m}$, there is no noticeable effect of the use of relays on the outage probability. This is because the BS sufficiently covers the whole cell by itself. In this region the use of relays increases the maximum number of served users. This is known as the throughput enhancement region.

Figure 7 shows the average number of G.711 users for different cell sizes when the system traffic load is set at $50 \%$. The average here is caclulated according to the MCSs distribution and corresponds to the most probable allocation of users within the cell. For a single-hop system, users close to the cell border are likely to be dropped and be replaced by users closer to the BS. On the other hand, the coverage 


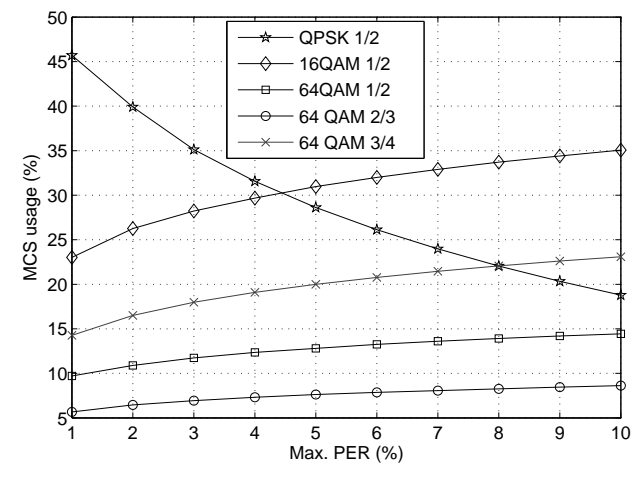

Figure 8. MCS usage vs. PER threshold in a non-relay cell

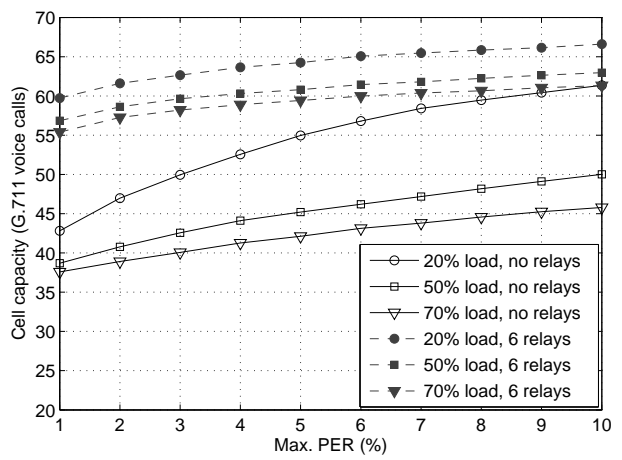

Figure 9. Max. number of voice calls in a cell vs. PER threshold

area for a relay-assisted system is larger but two hops may be used to serve far away users resulting in more consmed resources. As result a clear trade-off between capacity enhancement and coverage extension can be seen. This region is referred to as range extension.

It is now interesting to study how the choice of maximum PER, which defines MCS switching points, impacts the usage of the different modulation schemes within the cell. In Figure 8 , the usage of modulation schemes over maximum PER is depicted. Here, the cell size is fixed at $500 \mathrm{~m}$ resulting in the outage probability of $3 \%$. It is evident that a rise of maximum PER results in a decreased usage of QPSK 1/2 only while all other MCS are used more often.

The results of maximum VoIP users over the range of PER for a conventional cell as well as a relay-assisted cell with six relays are shown in Figure 9. It can be seen that when the traffic load from interfering cells is light, the system can accept more users into the victim cell. The use of relays clearly shows significant improvement in capacity.

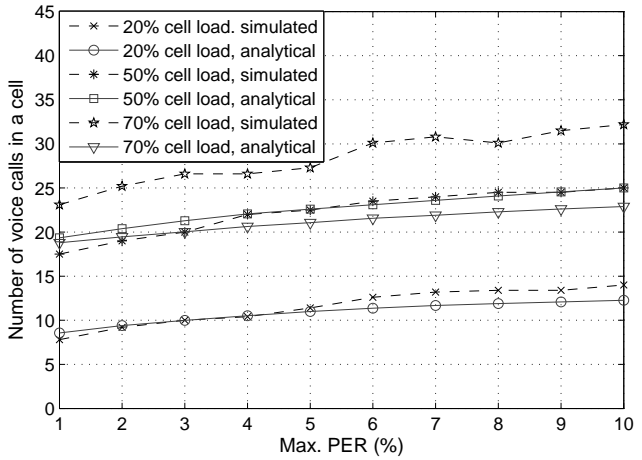

Figure 10. Analytical and simulated number of users in a non-relay cell

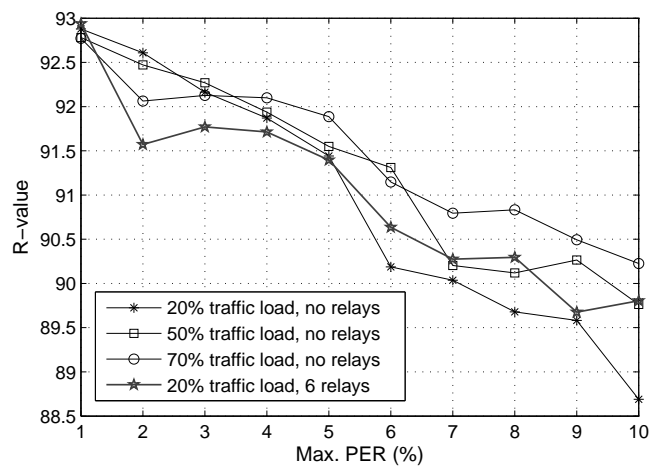

Figure 11. Average R-value among users in a cell vs. PER threshold

When no relay is used, the higher value of allowable PER results in a substantially higher number of supported users. However, the effect of changing the maximum PER is less evident when relaying is used because many users already use higher MCS via a relay and do not benefit from more aggressive MCS switching. In Figure 10 shows the average number of VoIP users per cell at different system traffic loads. The simulated results are obtained with a network simulator considering all overhead and protocol signaling in IEEE 802.16e.

Since the packet loss is one of the main factors that impact the transmitted voice quality, it is important to consider voice quality changes when the MCS allocation becomes more aggressive. The average R-value as a function of PER threshold is illustrated in Figure 11. The voice quality decreases with increasing maximum tolerable PER. However, it remains above value of 89 and can be described as "good" to "very good" [10]. It is shown that the voice quality on average does not depend on the level of interference as indi- 


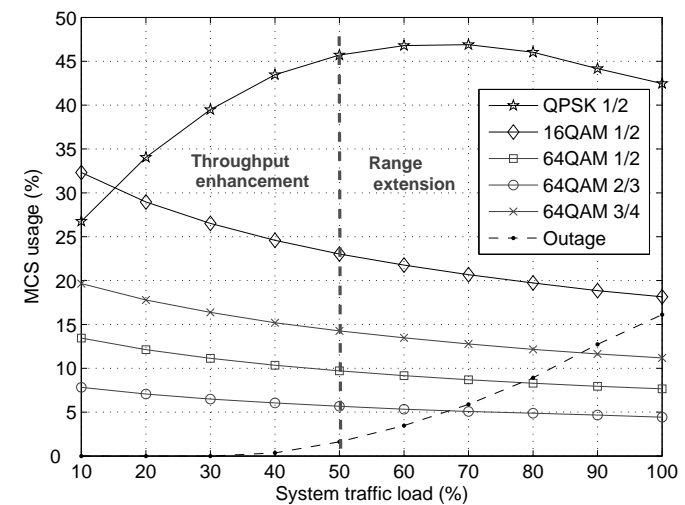

Figure 12. MCS usage in a non-relay cell vs. system traffic load

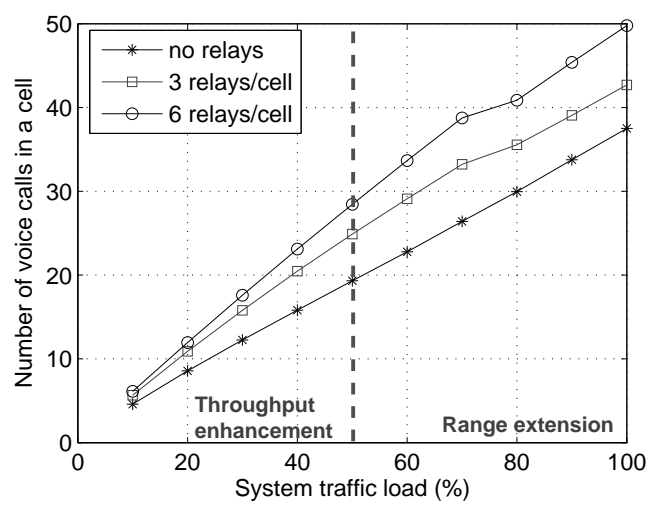

Figure 13. Voice calls vs. traffic load

cated by the traffic load. This is due to the fixed end-to-end PER threshold and low transmission delay in DL, since BW request access delay is not required.

Figure 12 illustrates the usage of different MCSs within the victim cell over the system traffic load. The traffic load increase and the corresponding decrease in SINR force users to switch to the most robust MCS, QPSK 1/2, or even to be dropped. Therefore, the use of relays can enable higher data rates for users who are far away from the base station or provide coverage where the most robust MCS can no longer be supported and thus increase the total system throughput and/or extend the coverage. Figure 13 illustrates the comparison of systems with none, three and six relays. The results show that the higher number of relays yields a better performance gain. However, interference, system complexity and overhead are critical in justifying the performance benefit of a large number of relays.

\section{Conclusion}

The impacts of multi-hop relaying on voice communication in the downlink OFDMA communication system are studied. We show that the system performance strongly depends on deployment and operating parameters such as size of the cell, traffic loads and the allowable packet error rate. In addition, the signaling overhead in deploying relay nodes plays an important role in determining the benefits of relayassisted techniques. Multi-hop relaying is most effective in highly loaded systems in reducing the outage probability while increasing the cell throughput.

\section{References}

[1] R. Pabst et al. Relay-based Deployment Concepts for Wireless and Mobile Broadband Radio. IEEE Commun. Mag., 42(9):80-89, September 2004.

[2] Y.-D. Lin and Y.-C. Hsu. Multihop Cellular: A New Architecture for Wireless Communication. In Proc. 2000 Conf. on Comp. Commun., pages 1273-1282, March 2000.

[3] N. Esseling, H. S. Vandra, and B. Walke. A Forwarding Concept for HiperLAN/2. Comput. Networks, 37(1):25-32, 2001.

[4] J. Cho and Z. Haas. On the Throughput Enhancement of the Downstream Channel in Cellular Radio Networks through Multihop Relaying. IEEE Jour. Sel. Areas Commun., 22(7):1206-1219, September 2004.

[5] N. Esseling, B. Walke, and R. Pabst. Performance of MACFrame-Based Protocols for Mobile Broadband Systems using Layer 2 Relays. In 11th Wireless World Research Forum, Oslo, Norway, June 2004.

[6] C. Cicconetti, A. Erta, L. Lenzini, and E. Mingozzi. Performance Evaluation of the IEEE 802.16 MAC for QoS Support. IEEE Trans. Mobile Comput., 6:26-38, 2007.

[7] H. Lee et al. Performance Analysis of Scheduling Algorithms for VoIP Services in IEEE 802.16e Systems. In Proc. 63rd IEEE VTC, volume 3, pages 1231- 1235, 2006.

[8] F. Hou, P.-H. Ho, and X. Shen. Performance evaluation for unsolicited grant service flows in 802.16 networks. In Proc. 2006 Int. Conf. on Commun. and Mobile Comput., pages 991-996, 2006.

[9] IEEE Std 802.16-2004. Standard for Local and Metropolitan Area Networks - Part 16: Air Interface for Fixed Broadband Wireless Access Systems, October 2004.

[10] ITU-T G.107. The Emodel, a computational model for use in transmission planning, 2005.

[11] WINNER II WP1. WINNER II Interim Channel Models. D1.1.1 v1.0, November 2006.

[12] IEEE 802.16j-06/026r3. Baseline for Air Interface for Fixed and Mobile Broadband Wireless Access Systems - Multihop Relay Specification, April 2007.

[13] IEEE Std 802.16e-2005. Amendment 2: Physical and Medium Access Control Layers for Combined Fixed and Mobile Operation in Licensed Bands, February 2005.

[14] ITU-T M.1225. Guidelines for evaluation of radio transmission technologies for imt-2000, 1997. 